\title{
A Single-Trial Estimation of the Feedback-Related Negativity and Its Relation to BOLD Responses in a Time-Estimation Task
}

\author{
Michael P. I. Becker, ${ }^{1,2}$ Alexander M. Nitsch, ${ }^{1}$ Wolfgang H. R. Miltner, ${ }^{1}$ and Thomas Straube ${ }^{1,2}$ \\ ${ }^{1}$ Department of Biological and Clinical Psychology, Friedrich Schiller University, D-07743 Jena, Germany, and ${ }^{2}$ Institute of Medical Psychology and Systems \\ Neuroscience, University Hospital Muenster, D-48149 Muenster, Germany
}

\begin{abstract}
An event-related potential (ERP) component reliably associated with feedback processing and well studied in humans is the feedbackrelated negativity (FRN), which is assumed to indicate activation of midcingulate cortex (MCC) neurons. However, recent approaches have conceptualized this frontocentral ERP component as reflecting at least partially a reward positivity associated with activation in reward-related brain regions, in line with $\mathrm{fMRI}$ studies investigating feedback processing in the context of reward evaluation. To discover convergence of electrophysiological and BOLD responses elicited by performance feedback, we concurrently recorded EEG and fMRI during a time-estimation task. The ERP showed relatively more negative amplitudes to negative than to positive feedback. Conventional analyses of fMRI data revealed activation of a number of areas, including ventral striatum, anterior cingulate cortex, and medial prefrontal cortex to positive versus negative feedback. Most importantly, when using single-trial amplitudes of electrophysiological feedback signals to estimate hemodynamic responses, we found feedback-related BOLD-responses in ventral striatum, midcingulate, and midfrontal cortices to positive but not to negative feedback associated with feedback signals in the time range of the FRN. Specifically, activation in these areas increased as amplitudes became more positive. These findings suggest that, in the time-estimation task, a positivity elicited by reward is associated with brain activation in several reward-related brain regions and is driving differential ERP responses in the time range of the FRN.
\end{abstract}

Key words: feedback-related negativity; fMRI; reward feedback; single-trial; time-estimation task

\section{Introduction}

Intact feedback processing informs adaptive behavior and has been shown to rely on frontal cortex (Rushworth et al., 2011). Well studied in humans is a negative deflection called the feedback-related negativity (FRN) in the averaged EEG timelocked to stimulus presentation, which was first described by Miltner et al. (1997) and has for more than a decade stimulated a lot of research as a neuronal index of unexpected negative feedback (Walsh and Anderson, 2012). Although not consistently (Müller et al., 2005; Nieuwenhuis et al., 2005; Carlson et al., 2011; Foti et al., 2011), dipole localization studies have converged on structures of the frontal midline, particularly the midcingulate cortex (MCC) (Vogt, 2009), as a possible generator of the FRN (Miltner et al., 1997; Gehring and Willoughby, 2002; Holroyd

\footnotetext{
Received Aug. 29, 2013; revised Jan. 10, 2014; accepted Jan. 14, 2014.

Author contributions: A.M.N. and T.S. designed research; A.M.N. performed research; M.P.I.B., A.M.N., and T.S. analyzed data; M.P.I.B., W.H.R.M., and T.S. wrote the paper.

This work was supported by the German Ministry of Education and Research (Bundesministerium für Bildung und Forschung, Grant 01GW0740). We thank Thomas Hiller, Carolin Sandrock, and Antonia Böhm for their contributions to data acquisition and analysis.

The authors declare no competing financial interests.

Correspondence should be addressed to Michael P. I. Becker, Institute of Medical Psychology and Systems Neuroscience, University Hospital Muenster, Von-Esmarch-Str. 52, D-48149 Muenster, Germany. E-mail: beckermi@unimuenster.de.

DOI:10.1523/JNEUROSCI.3684-13.2014

Copyright $\odot 2014$ the authors $\quad 0270-6474 / 14 / 343005-08 \$ 15.00 / 0$
}

and Coles, 2002; Ruchsow et al., 2002; Luu et al., 2003; Nieuwenhuis et al., 2004; Potts et al., 2006). Yet, evidence from neuroimaging studies on the role of MCC in performance feedback processing is mixed: some studies (Ullsperger and von Cramon, 2003; Holroyd et al., 2004) found the MCC to be more active during processing of negative feedback relative to positive feedback, whereas others reported no significant differences with regard to MCC (e.g., van Veen et al., 2004; Nieuwenhuis et al., 2005). However, several studies reported activation to positive relative to negative feedback in key regions of the reward circuit, in particular ventral (VS) and dorsal striatum (van Veen et al., 2004; Nieuwenhuis et al., 2005; Martin et al., 2009; Carlson et al., 2011).

Furthermore, there is increasing evidence that the FRN might result from superposition of a feedback-related positivity (FRP) upon the N2 and at least partly be driven by variance from rewarding feedback (Holroyd et al., 2008; Carlson et al., 2011; Foti et al., 2011). Moreover, recent work using EEG and fMRI has suggested that at least one additional neural generator of the FRN/FRP may lie within the striatum (Martin et al., 2009; Carlson et al., 2011; Foti et al., 2011), a structure difficult to investigate by scalp EEG (Cohen et al., 2011a).

We investigated the relation of feedback-related hemodynamic and electrophysiological indices by direct coupling and convergence estimation of EEG and fMRI data recorded simul- 
taneously during a time-estimation task known to reliably elicit a stable FRN/FRP. In particular, we combined data from both modalities to investigate in which brain regions BOLD signal changes are associated with a single-trial measure of the FRN/FRP (ST-FRN/FRP). We reasoned that the degree of condition-specific coupling of ST-FRN/FRP should be amplified during positive trials as implied by more recent studies of the differential processing of positive and negative feedback. Further, we expected to find activation of the cingulate cortices to be coupled to STFRN/FRP as the FRN has repeatedly been reported to be associated with activation of cingulate structures (Walsh and Anderson, 2012). Last, we hypothesized that, if the FRN/FRP indexes reward-related feedback processing, its trial-by-trial variability should be coupled to activation of central structures of the reward circuit, foremost VS.

\section{Materials and Methods}

Participants. A total of 25 persons ( 9 females; mean age $25.6 \pm 7.7$ years) with no known history of neurological or psychiatric disease participated in this study for payment or course credit. The study was approved by the Ethics Committee of the University of Jena.

Of the 25 recruited healthy participants, data from 15 participants fulfilled all criteria for the ST-FRN/FRP informed general linear modeling (GLM) analysis: data from three participants were discarded because of poor EEG quality resulting from strong artifact contamination; data from one participant were discarded because EEG markers were not correctly written to file; data from one person were excluded because of excessive motion artifact as estimated by a 3D head motion correction algorithm as described below; data from five additional participants were excluded because no reliable single-trial or grand average FRN/FRP could be obtained for statistical purposes.

Stimuli and task. The time-estimation paradigm underlying the present study required participants to estimate an interval of $1 \mathrm{~s}$ as accurately as possible (Fig. 1). An auditory cue indicating the onset of each timeestimation trial was presented for $50 \mathrm{~ms}$. Participants were required to press a button with their right index finger just when they thought that $1 \mathrm{~s}$ had elapsed. During auditory cue presentation and for the following 4200-7600 ms (depending on a random stimulus onset asynchrony [SOA]; see below), a white fixation cross on a black background was presented centrally. Feedback was administered visually after offset of fixation cross presentation and consisted of either of the letters A, B, or C, which were presented for $1 \mathrm{~s}$, respectively. Each of these letters was assigned to one of three feedbacks: it indicated a correct response, a false response, or did not inform subjects about the adequacy of their time estimation. The specific assignment of letters to conditions was randomized across subjects. All stimuli were projected onto a screen inside the scanner bore. Every subject was given the opportunity to practice the task in an online training run of 9 trials before which the sound volume of the auditory cues was adjusted to the subject's individual specifications. Then, every subject completed two runs of 66 trials, respectively.

To decorrelate response- and stimulus-related activation patterns, SOA and intertrial intervals were systematically jittered (Miezin et al., 2000). Specifically, SOA intervals were offset after button presses by a value randomly chosen from the range $4200-7600 \mathrm{~ms}$; intertrial intervals were offset by a value randomly chosen from the range 3200-7500 ms. Furthermore, a sliding response deadline algorithm was used to balance the relative presentation frequency of the three different feedback types. To this end, a time window centered $\sim 1 \mathrm{~s}$ after cue presentation, the target time point, was defined. The training run was used to establish an individual baseline of this time window's length for every subject. In the experiment proper, this baseline was used as the starting value and ad- justed trialwise according to the following criteria: in case of an insufficiently accurate response, the window is widened by $20 \mathrm{~ms}$; in case of an accurate response, the window is shortened by $20 \mathrm{~ms}$. The ratio of ambiguous feedback is fixed to exactly 22 presentations per run (i.e., $33 \%$ ).

The response deadline is an important factor in the time-estimation task as it prevents subjects from building up strong expectations about the information conveyed by feedback. That is, because the local probability of a quickly learned behavioral response being sufficiently accurate is adapted trialwise depending on the individuals' accumulated performance levels, habit formation is unlikely to take place quickly. Thus, the response situation remains underdetermined throughout the experiment, and feedback presentation is needed to clarify the response outcome.

Postexperimentally, subjects rated valence and arousal of feedback on 9-point Likert scales ( 1 = "not at all"; 9 = "very much"). In one participant, these rating data are missing due to a technical error.

fMRI data acquisition. fMRI data were acquired at $3 \mathrm{~T}$ on a Siemens Magnetom Trio (Siemens Medical Systems) using a standard 12-channel Siemens Head Matrix Coil. Simultaneously, EEG data from 63 scalp electrodes were recorded.

Two runs of 376 volumes, each consisting of 35 slices (slice thickness $=$ $3 \mathrm{~mm}$; interslice gap $=0.50 \mathrm{~mm}$; in-plane resolution $=3 \times 3 \mathrm{~mm}^{2}$, interslice time $=60 \mathrm{~ms}$ ) were recorded by means of a T2*-weighted gradient-echo, echoplanar sequence with a repetition time (TR) of 2300 $\mathrm{ms}$, an echo time (TE) of $30 \mathrm{~ms}$, and a flip angle (FA) of $90^{\circ}$, yielding a data matrix of $64 \times 64$ voxels within a field of view (FOV) of $192 \mathrm{~mm}$. The TR included a silent interval of $200 \mathrm{~ms}$ with scanner gradient fields turned off, which allowed for visual monitoring of proper EEG recording and improved baseline estimation in the subsequent analyses (Debener et al., 2005, 2007). Additionally, a T1-weighted MPRAGE structural volume in either high- (192 slices) or low-resolution (96 slices) was recorded for anatomical localization. To minimize external magnetic field inhomogeneities, a shimming field was applied before functional imaging.

EEG data acquisition. Concurrent EEG data were recorded from 63 sintered $\mathrm{Ag} / \mathrm{AgCl}$ ring electrodes mounted within an MRI-compatible cap (BRAINCAP-MR, EASYCAP) fitted on the scalp so that 21 corresponded to the international 10-20 system and the remaining were interspaced equally into this system. Two additional electrodes were placed to monitor electrocardiac activity at the left-side back (between costae 5 and 7) and electro-ocular activity below the left eye, respectively. Electrode gel (Abralyt, 2000, EASYCAP) was applied to electrode-scalp junctions until electrode impedances fell within the range of $0-5 \mathrm{k} \Omega$. A BrainAmp-MR amplifier (BrainProducts) was fixed inside the scanner bore and connected to a PC in the console room via fiber-optic cable, where the EEG was recorded with a sampling rate of $5000 \mathrm{~Hz}$, an online bandpass filter of $0.016-250 \mathrm{~Hz}$, the recording reference at frontocentral electrode FCz, and the ground at electrode Iz. The BrainProducts SyncBox was used to synchronize the scanner gradient and EEG acquisition system clocks.

Preprocessing of fMRI data. Preprocessing and analysis of the functional data were performed using Brain Voyager QX 1.10 and Brain 


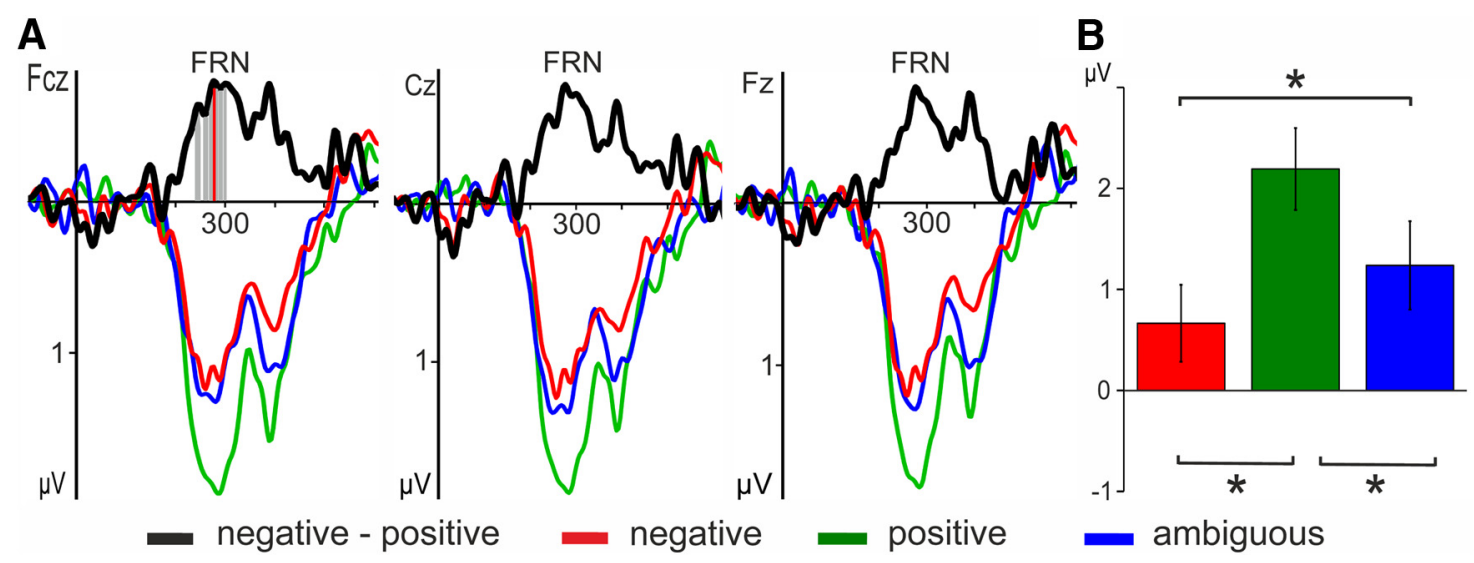

Figure 2. A, Grand average ERPs at FCz (left), $\mathrm{Cz}$ (middle), and Fz (right) for the three conditions: negative (red), positive (green), and ambiguous (blue), as well as the difference wave (black). Gray bins represent latency dispersions in the location of single-subject peaks (85th percentile latency range of individual peaks); the bold red line indicates the time point of the grand average FRN. $B$, Means ( \pm SEM) of the grand average ERPs at FCz for each of the three conditions. *Significant $t$ values.

A

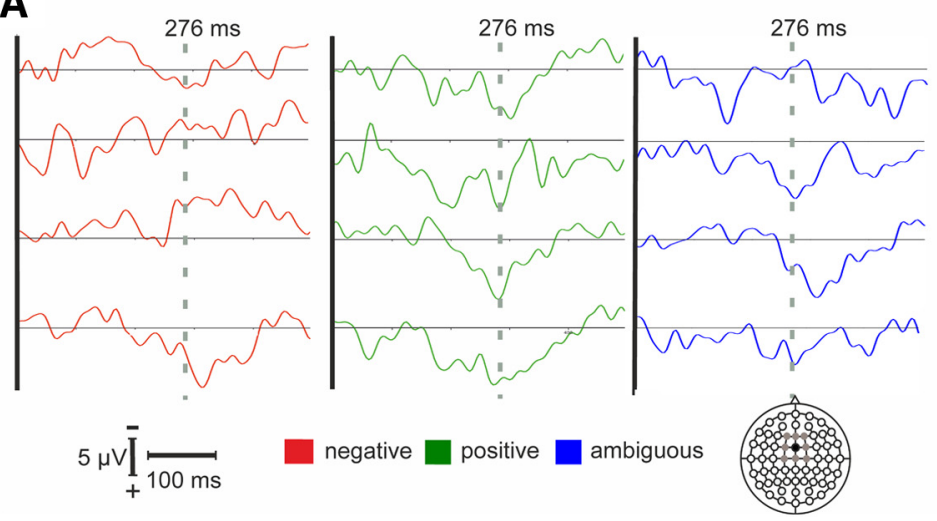

B

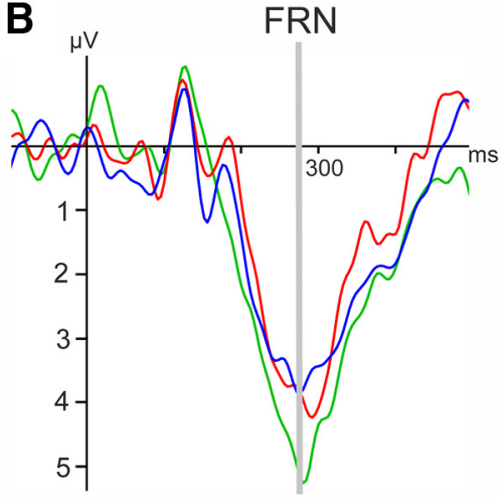

Figure 3. A, Parameterization of the single-trial FRN/FRP. Four denoised single trials for each condition of one single subject are shown at electrode FCz. $\boldsymbol{B}$, ERP averaged over all trials of the same subject as in $\boldsymbol{A}$ for all three conditions: negative (red), positive (green), and ambiguous (blue).

Voyager QX 2.4 software (BVQX; Brain Innovation). The first four volumes of each run were discarded as dummies to ensure steady-state tissue magnetization. Realignment to the first volume of each run was performed via least-squares estimation of six rigid body parameters to reduce effects of head movements on volume time course analysis. Further data preprocessing comprised a correction for slice time errors and spatial (8 mm FWHM isotropic Gaussian kernel) as well as temporal (high pass filter: 8 cycles per run; low pass filter: $2.8 \mathrm{~s}$ FWHM; Linear Trend Removal) smoothing. Anatomical and functional images were coregistered and normalized to the Talairach space (Talairach and Tournoux, 1988).

Preprocessing of EEG data. Algorithms following procedures proposed by Allen et al. $(1998,2000)$ were used as implemented in BrainVisionAnalyzer 2.0 Software (Brain Products) to correct the EEG data for gradient and pulse artifacts. Eye movement-related artifacts were removed using a dipole source modeling procedure in BESA 5.2 (BESA). The signal time course at $\mathrm{FCz}$ was reconstructed from the signals of the adjacent electrodes (F1, Fz, F2, FC1, FC2, C1, Cz, C2) by topographic interpolation. An averaging procedure that reconstructs the signal time course from several electrodes should be more resilient toward interindividual variability in FRN topography. As has been shown before (Amiez et al., 2013), a great deal of morphological variability in individual gyrification can be expected in feedback presentation tasks, and this variability also influences electrophysiological parameters (Huster et al., 2009). Therefore, we reconstructed the signal time course from several electrodes adjacent to $\mathrm{FCz}$ to minimize the risk of identifying secondorder potentials (Fig. $2 A$ ).

Subsequently, EEG data were high pass-filtered at $0.1 \mathrm{~Hz}$, low passfiltered at $30 \mathrm{~Hz}$, downsampled to $250 \mathrm{~Hz}$, and rereferenced to common average. The signal time course was decomposed into maximally independent components (ICs) by the extended biased infomax algorithm as implemented in BrainVisionAnalyzer's Independent Component Analysis transformation based on $10 \mathrm{~s}$ interval trial markers. Components with frontocentral topography and maximum amplitude difference wave between positive and negative feedback in the time range of $180-300 \mathrm{~ms}$ in the backprojected trial-averaged event-related potentials (ERPs) were chosen for backprojection to isolate the ST-FRN/FRP from processes of no interest and thus increase signal-to-noise-ratio (Fig. 2A; see 4A). An average of 2.5 components per subject (SD 1.2) were backprojected. The signal time course of each trial and electrode was segmented into $600 \mathrm{~ms}$ epochs with the mean signal time course from $-100 \mathrm{~ms}$ to stimulus onset serving as baseline.

Predictors and contrasts of interest. The statistical analysis of fMRI data was based on the GLM with adjustment for autocorrelation following a global AR(1) model. Because it is unclear whether the FRP is exactly symmetrical in time to the FRN, single-trial weights used in the parametric $\mathrm{fMRI}$ analysis were defined as follows: within the defined search interval, at the time point of the maximum difference $t$-score of the contrast between positive and negative feedback EEG signal time courses at FCz the amplitude in each trial served as ST-FRN/FRP (to establish correspondence with the terminology of other studies investigating the FRN/ FRP as FRN, weights were negated, i.e., greater weights correspond to a "more negative" FRN; Fig. $3 A, B$ ). This approach relates to both concepts interchangeably and establishes correspondence between the grand average and single-trial literature. The expected BOLD signal change for each predictor was modeled by convolving the event reference functions with a 2-gamma hemodynamic response function (HRF); and in the case 
A
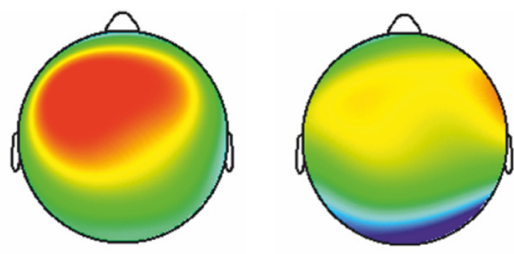

B

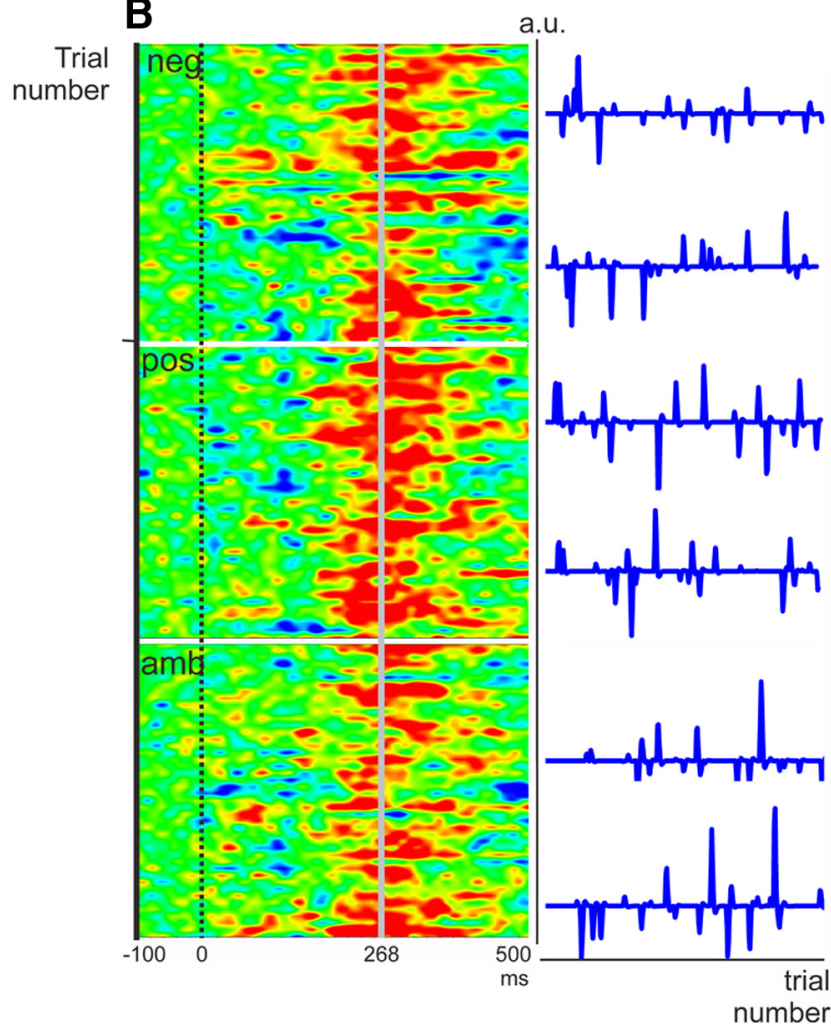

Figure 4. Extraction of the single-trial FRN/FRP and construction of the hemodynamic response functions. $\boldsymbol{A}$, Example of frontocentral ICs selected for backprojection in one subject. $\boldsymbol{B}$, Stacked plots of all trials in the same subject as in $\boldsymbol{A}$ ordered by feedback condition; gray line indicates point of grand average maximum difference wave (left column). Parametric hemodynamic response functions extracted from these trials (right column).

of feedback predictors, these HRFs with the respective ST-FRN/FRP weights. Using the same set of HRFs, two GLMs were modeled, both of which comprised predictors for auditory cue presentations, missed trials, and unmodulated feedback as well as ST-FRN/FRP-modulated feedback. The two GLMs differed only in the way the feedback predictors were implemented: specifically, the first GLM comprised a unified feedback predictor across all three feedback conditions consisting of HRFs with unit height and its ST-FRN/FRP-modulated version (i.e., we convolved the stimulus-locked feedback-related HRFs of all three conditions with the ST-FRN/FRP amplitudes into one predictor) (Debener et al., 2005). We refer to this GLM as the across conditions model (ACM). The ACM was modeled to address the question in which voxels BOLD signal changes were associated with changes in FRN/FRP amplitude and estimates the degree of trial-by-trial coupling. The second GLM comprised separate predictors for the three feedback categories (positive, negative, and ambiguous) supplemented by the three ST-FRN/FRP-modulated feedback predictors for these categories (Fig. 4B). We refer to this model as the condition-specific model (CSM). The intention behind the CSM was to investigate potential condition-specific coupling. Beta-weights for the GLMs were determined by a least-squares estimation under consideration of random effects.

A cluster-size threshold estimation procedure was used (Forman et al., 1995; Goebel et al., 2006) to correct for multiple comparisons. Significant

Table 1. Coordinates of activation clusters identified in the conventional random effects analysis $^{a}$

\begin{tabular}{|c|c|c|c|c|c|c|}
\hline \multirow[b]{2}{*}{ Region } & \multirow[b]{2}{*}{ Lateralization } & \multicolumn{3}{|c|}{$\begin{array}{l}\text { Talairach } \\
\text { coordinates }\end{array}$} & \multirow{2}{*}{$\begin{array}{l}\text { Cluster } \\
\text { size }\left(\mathrm{mm}^{3}\right)\end{array}$} & \multirow[b]{2}{*}{ Fvalue } \\
\hline & & $x$ & $y$ & $z$ & & \\
\hline \multicolumn{7}{|l|}{ Conventional model } \\
\hline \multirow[t]{2}{*}{ Ventral striatum } & L & -12 & 2 & -2 & 14877 & 39.7 \\
\hline & $\mathrm{R}$ & 24 & 20 & -2 & 11394 & 23.2 \\
\hline Anterior cingulate cortex & $\mathrm{L}$ & -3 & 32 & 13 & 14472 & 17.6 \\
\hline Inferior frontal gyrus & L & -51 & 41 & 4 & 3132 & 19.2 \\
\hline Insula & $\mathrm{L}$ & -33 & 20 & 4 & 6129 & 21.5 \\
\hline \multirow[t]{2}{*}{ Claustrum } & L & -33 & 11 & -2 & 324 & 18.5 \\
\hline & L & -27 & -4 & 16 & 297 & 9.1 \\
\hline \multirow[t]{2}{*}{ Superior temporal gyrus } & $\mathrm{R}$ & 48 & -52 & 19 & 9342 & 15.7 \\
\hline & L & -51 & -58 & 19 & 5481 & 16.5 \\
\hline Precuneus & $\mathrm{R}$ & 6 & -58 & 31 & 7479 & 24.4 \\
\hline Posterior cingulate & L & -12 & -58 & 10 & 1782 & 10.5 \\
\hline \multirow[t]{2}{*}{ Superior frontal gyrus } & $\mathrm{R}$ & 6 & 50 & 43 & 243 & 8.3 \\
\hline & L & -27 & 29 & 52 & 459 & 7.9 \\
\hline \multirow[t]{2}{*}{ Middle frontal gyrus } & L & -27 & 17 & 46 & 324 & 7.9 \\
\hline & $\mathrm{R}$ & 33 & 65 & 13 & 999 & 10.8 \\
\hline Middle temporal gyrus & $\mathrm{R}$ & 57 & -16 & -8 & 1917 & 12.4 \\
\hline
\end{tabular}

a Peak coordinates of activation clusters obtained at a threshold of $p<0.005$, uncorrected, and cluster size $\geq 1$ voxels $\left(297 \mathrm{~mm}^{3}\right)$.

clusters of contiguously activated voxels were determined by a Monte Carlo simulation based on 5000 iterations. After setting the cluster-level false positive-rate to $p<0.005$ (uncorrected), the simulation resulted in a minimum cluster size of 11 contiguously activated voxels corresponding to a false-positive rate of $5 \%$. An a priori-defined whole-brain mask was used to mask out nonbrain tissue, including the ventricles. The watershed-algorithm of Neuroelf (v0.9c; http://neuroelf.net/; i.e., the splitclustercoords function) was used to assess local maxima of clusters.

\section{Results}

\section{Behavioral results}

On average, participants received $32.4 \%$ positive (SEM $\pm 0.6 \%$ ), $33.0 \%$ negative (SEM $\pm 0.8 \%$ ), and $32.7 \%$ ambiguous (SEM $\pm 0.4 \%$ ) feedback. Presentation frequencies did not differ significantly between feedback conditions $\left(F_{(11.1,14.7)}=0.28 ; p=\right.$ 0.617).

Subjects' ratings differed between feedback categories for valence $\left(F_{(2,26)}=82.6, p<0.005\right)$ and arousal $\left(F_{(2,26)}=7.6, p<\right.$ $0.005)$. In particular, negative feedback was rated more arousing $\left(t_{(13)}=3.2, p<0.01\right)$ and less positive $\left(t_{(13)}=-3.3, p<0.01\right)$ than ambiguous feedback, whereas positive feedback was rated more positive than negative $\left(t_{(13)}=10.2, p<0.005\right)$ and ambiguous feedback $\left(t_{(13)}=13.5, p<0.005\right)$ and more arousing than ambiguous feedback $\left(t_{(13)}=3.0, p<0.05\right)$.

\section{fMRI results}

In conventional analyses, we addressed differences between the nonparametric feedback predictors by BVQX's ANOVA function with feedback condition as within-subjects factor (see Table 1 for a comprehensive overview of local maxima in whole-brain activation and Fig. 5A). Most notably, activation in VS (peak $x, y, z$ : $-12,2,-2 ; F_{(2,28)}=39.7, p<0.05$, corrected; Fig. $\left.5 B\right)$ and anterior cingulate areas (ACC; peak $x, y, z:-3,32,13 ; F_{(2,28)}=$ $17.6, p<0.05$, corrected) differed significantly between the three conventional, nonparametric feedback predictors. Moreover, activation was found in posterior cingulate cortex (PCC), insular cortex, medial and superior frontal gyri (SFG), as well as in temporal regions and precuneus. Post hoc $t$ tests for activations of the ventral striatal and anterior cingulate clusters indicated that these regions were more responsive to positive feedback compared 


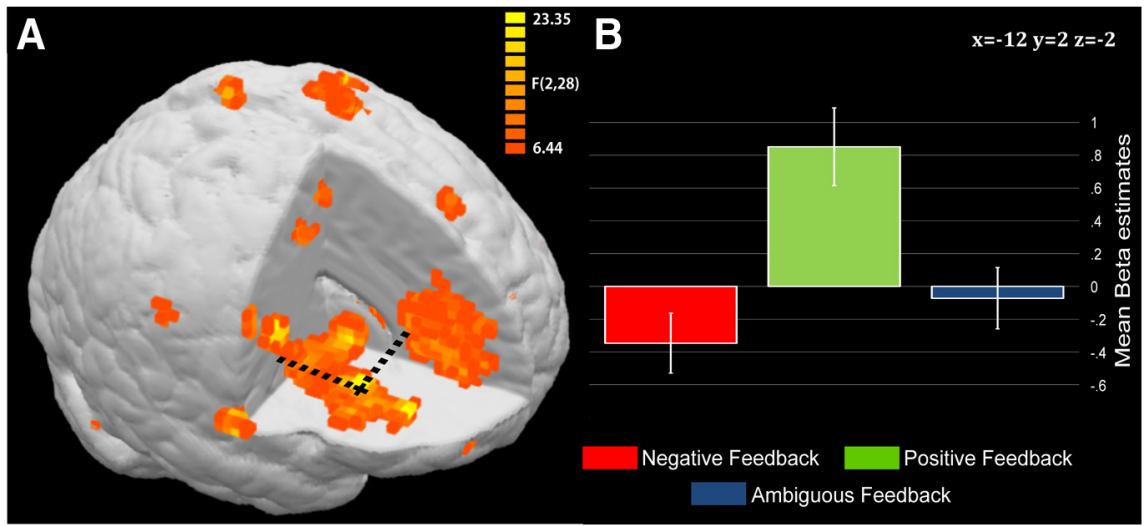

Figure 5. Result of the conventional, nonparametric GLM analysis. $\boldsymbol{A}$, F-Map of feedback condition differences for the conventional random-effects analysis with positive, negative, and ambiguous feedback as factor levels. $\boldsymbol{B}$, F-Map beta estimates ( \pm SEM) for all three conditions in ventral striatal cluster. Whole-brain cluster corrected (11 voxels at $p<0.005$ ); 13 voxels projection depth.

Table 2. Coordinates of activation clusters identified in the EEG-informed parametric analyses $^{a}$

\begin{tabular}{|c|c|c|c|c|c|c|}
\hline \multirow[b]{2}{*}{ Region } & \multirow[b]{2}{*}{ Lateralization } & \multicolumn{3}{|c|}{ Talairach coordinates } & \multirow{2}{*}{$\begin{array}{l}\text { Cluster } \\
\text { size } \\
\left(\mathrm{mm}^{3}\right)\end{array}$} & \multirow[b]{2}{*}{$t$ value } \\
\hline & & $x$ & $y$ & $z$ & & \\
\hline \multicolumn{7}{|l|}{ Across conditions model } \\
\hline \multirow[t]{2}{*}{ Middle temporal gyrus } & $\mathrm{R}$ & 60 & -49 & -14 & 2214 & -6.3 \\
\hline & $\mathrm{L}$ & -57 & -37 & -11 & 1215 & -5.3 \\
\hline Inferior frontal gyrus/claustrum & $\mathrm{R}$ & 27 & 35 & -5 & 1512 & -5.6 \\
\hline Ventral striatum & L & -12 & 5 & -2 & 945 & -5.1 \\
\hline Inferior temporal gyrus & $\mathrm{R}$ & 45 & -43 & -20 & 675 & -5.1 \\
\hline Parahippocampal gyrus & $\mathrm{R}$ & 24 & -13 & -26 & 621 & -5.2 \\
\hline Medial frontal gyrus & $\mathrm{R}$ & 9 & 41 & 43 & 576 & -4.0 \\
\hline \multirow[t]{2}{*}{ Cingulate cortex } & $\mathrm{R}$ & 6 & 38 & 16 & 486 & -4.3 \\
\hline & $\mathrm{L}$ & -6 & 35 & 25 & 432 & -4.8 \\
\hline Precentral gyrus & $\mathrm{L}$ & -42 & -1 & 22 & 486 & -4.3 \\
\hline Posterior cingulate & $\mathrm{R}$ & 21 & -31 & 49 & 378 & -4.1 \\
\hline
\end{tabular}

a Peak coordinates of activation clusters obtained at a threshold of $p<0.005$, uncorrected, and cluster size $\geq 11$ voxels $\left(297 \mathrm{~mm}^{3}\right)$.

with negative feedback (VS: $t_{(14)}=6.9, p<0.05$, corrected; ACC: $t_{(14)}=5.0, p<0.05$, corrected) and ambiguous feedback (VS: $t_{(14)}=5.9, p<0.05$, corrected; ACC: $t_{(14)}=4.3, p<0.05$, corrected), respectively. None of these regions, however, responded more strongly to negative feedback relative to ambiguous feedback (all, $p>0.05$, corrected). The only regions responding stronger to negative feedback than positive feedback were found in PCC, and insula (all, $t>3.8$; all, $p<0.05$, corrected). A conjunction analysis of [(positive feedback $>$ baseline) AND (negative feedback $>$ baseline)] (Nieuwenhuis et al., 2005) revealed that MCC was activated by positive and negative feedback to a similar degree as a large cluster in MCC extending into the presupplementary motor area (first peak $x, y, z: 9,17,34, t_{(14)}=$ 5.8; second peak $x, y, z:-9,20,32, t_{(14)}=4.5$; third peak $x, y, z: 0$, $2,55, t_{(14)}=5.9$; cluster size $17,523 \mathrm{~mm}^{3}$; all, $p<0.05$, corrected) was significantly active in both contrasts.

\section{ERP results}

A repeated-measures ANOVA revealed a main effect of feedback condition $\left(F_{(2,28)}=25.5, p<0.05\right)$. Post hoc comparison of peak amplitudes at $\mathrm{FCz}$ across conditions by means of paired $t$ tests revealed significantly larger (i.e., more negative) FRN/FRPamplitudes for negative (mean $0.66 \mu \mathrm{V}$ ) relative to positive (mean $2.19 \mu \mathrm{V})$ feedback $\left(t_{(14)}=9.2, p<0.05\right.$ ), for ambiguous (mean $1.24 \mu \mathrm{V})$ relative to positive feedback $\left(t_{(14)}=4.1, p<\right.$
$0.05)$, and for negative relative to ambiguous feedback $\left(t_{(14)}=2.4, p<0.05\right.$; Fig. $2 B)$.

\section{EEG-informed fMRI results}

First, we examined the extent of trial-bytrial coupling of BOLD and ST-FRN/FRP signatures across all feedback conditions in the ACM. Parametric modulations of the FRN/FRP were (negatively) associated with voxel signal changes across the cingulate cortices, specifically in ACC (peak $x, y, z: 6,38,16, t_{(14)}=-4.3, p<0.05$, corrected), MCC (peak $x, y, z:-6,35,25$, $t_{(14)}=-4.0, p<0.05$, corrected $)$, and PCC (peak $x, y, z: 21,-31,49, t_{(14)}=$ $-4.1, p<0.05$, corrected). Moreover, voxels within the ventral striatum revealed strong coupling to the ST-FRN/ $\operatorname{FRP}\left(\right.$ peak $x, y, z:-12,5,-2, t_{(14)}=-5.1, p<0.05$, corrected). Further, we found the ST-FRN/FRP coupled to BOLD responses in medial frontal gyrus (MFG), inferior frontal gyrus, middle and inferior temporal gyrus, parahippocampal gyrus, and precentral gyrus (Table 2; Fig. 6A).

Second, we analyzed in which voxels a significant coupling of single-trial BOLD and FRN/FRP would be found conditionwise in the CSM. We therefore investigated the degree of coupling between ST-FRN/FRP and voxel signal changes for each feedback condition separately. Using this approach, it was evident that coupling found in the ACM was most pronounced during positive feedback while being diminished during negative and ambiguous feedback (Fig. 6B). In the positive feedback condition, ST-FRN/FRP-coupled activation was carried by voxels in VS (first peak $x, y, z: 3,8,-2, t_{(14)}=-5.1, p<0.05$, corrected; second peak $x, y, z:-9,11,-2, t_{(14)}=-4.8, p<0.05$, corrected), MFG (peak $x, y, z: 12,47,4, t_{(14)}=-5.1, p<0.05$, corrected), MCC (first peak $x, y, z: 6,17,28, t_{(14)}=-4.4, p<$ 0.05 , corrected; second peak $x, y, z: 0,35,28, t_{(14)}=-5.3, p<$ 0.05 , corrected), and ACC (peak $x, y, z: 6,32,-5, t_{(14)}=-4.3$, $p<0.05$, corrected) among several other structures (Table 3 ). In all regions, the association between BOLD responses and ERPs indicates that BOLD responses increase with more positive amplitudes (and/or decrease with more negative amplitudes).

In the negative condition, even at the most liberal thresholds, only two clusters coupled to ST-FRN/FRP were identifiable, both of which were located subgyrally (first peak $x, y, z:-21,-10,37$, $t_{(14)}=-5.44, p<0.05$, corrected; second peak $x, y, z:-24,17$, $25, t_{(14)}=-4.91, p<0.05$, corrected). In the ambiguous condition, no clusters survived the correction.

\section{Discussion}

By merging data from concurrently recorded EEG and fMRI to estimate convergence of electrophysiological and BOLD responses elicited by feedback on the single-trial level, we identified brain regions in which BOLD signal change was explained by trial-to-trial variability of the FRN/FRP. Most notably, our results reveal that hemodynamic activity within multiple sites in the frontal midline and the basal ganglia accompanies gradations of the electrophysiological signature of the FRN/FRP, as we found coupling between EEG and fMRI data in MCC, MFG, and VS, with increased BOLD responses associated with more positive ERP amplitudes. Importantly, additional analysis showed that ST-FRN/FRP variance explained the BOLD signal time course to 
positive, but not to negative, feedback in ACC, MFG, and VS, implying a contribution of these areas to the generation of the FRN/FRP.

\section{Midline regions associated with the FRN/FRP}

Many dipole localization studies have located plausible generators of the FRN/ FRP within the ACC and MCC (Walsh and Anderson, 2012). Other proposals highlighted interactions between SFG and PCC (Müller et al., 2005; Nieuwenhuis et al., 2005) and the insula (Doñamayor et al., 2011). Yet, as dipole modeling algorithms are not optimally suited to investigate multiple source solutions, the question of which structures contribute to scalp topography and source estimates of the FRN/FRP calls for alternative approaches to network characterization (Yao and Dewald, 2005).

Previous reports have rarely addressed divergences between source-localization results. Yet, different approaches in paradigms and imaging modalities yield different estimates for sources quintessentially or epiphenomenally related to the FRN/ FRP. We present data suited to bridge the gap between different approaches. Our data imply contributions of multiple frontal midline generators to elicitation of feedback-related BOLD and ERP signatures and corroborate in particular a role of MCC/ACC in FRN/FRP generation (Walsh and Anderson, 2012). Furthermore, coupling was also observed within a second, more dorsal medial region of medial prefrontal cortex (MPFC), and in ventral medial prefrontal cortex (VMPFC). Remarkably, in all these regions, activation was coupled to a relative positivity in the time range of the ST-FRN/FRP. Intracerebral recordings in human cingulate cortex have also found the same pattern of activation, with neurons in MCC responding stronger to positive than to negative feedback (Jung et al., 2010).

Furthermore, results from the CSM identified a cluster in ACC extending into VMPFC, which tracked amplitude modulations of the ST-FRN/FRP during positive feedback trials. In accordance with the literature (Levy and Glimcher, 2012), the effect in this cluster can be assumed to represent value mappings during receipt of reward as activation in VMPFC appears to reflect a common currency representation of rewarding stimuli.

\section{Striatal contributions to FRN/FRP generation}

Information about the neural generators of the FRN/FRP stems from scalp map topographies and dipole modeling studies. In the past, mass activation of cingulate neurons has been most often identified as a plausible generator of the FRN/FRP. Carlson et al. (2011) expanded this finding by presenting evidence of striatal contributions to FRN/FRP generation. They were the first to report a positive correlation between grand average FRN/FRP amplitude and activation within VS and MPFC. Our findings corroborate this association, as we found striatal and MPFC BOLD signatures to be explained by the ST-FRN/FRP. This finding lends further credence to the assumption that the FRN/FRP indexes the neuronal correlates of reward processing in frontostriatal networks, as implied by the extensive literature on the neuroscience of reward and punishment processing (e.g., Haber and Knutson, 2010) and the findings of Carlson et al. (2011). The ventral striatum, and more specifically the nucleus accumbens,
Table 3. Coordinates of activation clusters separately for each feedback condition ${ }^{a}$

\begin{tabular}{|c|c|c|c|c|c|c|}
\hline \multirow[b]{2}{*}{$\operatorname{Reg}$} & \multirow[b]{2}{*}{ Lateralization } & \multicolumn{3}{|c|}{ Talairach coordinates } & \multirow{2}{*}{$\begin{array}{l}\text { Cluster } \\
\text { size } \\
\left(\mathrm{mm}^{3}\right)\end{array}$} & \multirow[b]{2}{*}{$t$ value } \\
\hline & & $x$ & $y$ & $z$ & & \\
\hline
\end{tabular}

Positive feedback in the condition-specific model

Anterior cingulatecotex

$L$
$R$
$R$

Anterior cingulate cortex $\quad R$

Cingulate gyrus

$R$
$L / R$

Medial frontal gyrus

Inferior frontal gyrus

Insula

Postcentral gyrus

Inferior occipital gyrus

Lingual gyrus

Superior temporal gyrus

Parahippocampal gyrus

Precuneus

Posterior cingulate

Superior frontal gyrus

Middle frontal gyrus

\begin{tabular}{rrrrr}
-9 & 11 & -2 & 1080 & -4.8 \\
3 & 8 & -2 & 2241 & -5.1 \\
6 & 32 & -5 & 378 & -4.3 \\
0 & 35 & 28 & 2322 & -5.3 \\
6 & 17 & 28 & 459 & -4.4 \\
12 & 47 & 4 & 1269 & -5.1 \\
-18 & 32 & -8 & 675 & -5.8 \\
-39 & 8 & -8 & 783 & -5.3 \\
24 & -34 & 49 & 1809 & -5.7 \\
27 & -88 & -14 & 1512 & -5.4 \\
-9 & -94 & -5 & 3807 & -6.2 \\
48 & -16 & -20 & 12015 & -8.6 \\
-24 & -16 & -20 & 3456 & -6.8 \\
-3 & -40 & 49 & 513 & -4.4 \\
9 & -46 & 25 & 2808 & -5.8 \\
27 & 20 & 55 & 810 & -4.4 \\
-42 & 11 & 37 & 1971 & -5.6 \\
30 & 20 & 34 & 3618 & -4.7 \\
-36 & 50 & 7 & 1620 & -5.3 \\
\hline
\end{tabular}

a Peak coordinates of activation clusters obtained at a threshold of $p<0.005$, uncorrected, and cluster size $\geq 11$ voxels $\left(297 \mathrm{~mm}^{3}\right)$.

represents a structure that is an integral part of the so-called reward circuit; consequently, its activation is most often reported in the context of reward (Liu et al., 2011). If the FRN is indeed a reward positivity, coupling of the electrophysiological scalp signatures and the BOLD signal time course of the ventral striatum should be positively associated with the magnitude of amplitudes and reliably detectable specifically during positive feedback. Our results satisfy this requirement. Our findings are difficult to reconcile with theories, which assume that the variance of the FRN/FRP is generated in negative feedback trials, at least under the current protocol. Rather, we found that a hemodynamic signal coding feedback processing is amplified during positive trials and, furthermore, that this signal is carried by the VS, MPFC, and cingulate cortex.

Interactions of basal ganglia and cingulate cortices have been proposed in the EEG literature as main contributors to the FRN/ 
FRP (Holroyd and Coles, 2002), but closed-loop architecture of striatal neurons may restrict their role in FRN/FRP elicitation (Cohen et al., 2011a). Therefore, we cannot infer from our data that an FRN generator is necessarily located within VS. However, coupling of hemodynamic and electrophysiological approaches clearly implies an at least modulating contribution of VS to FRN/ FRP scalp maps, possibly via synchronization of VS and medial frontal, including cingulate regions as suggested by current models (Cohen et al., 2011b). Lesion studies might be helpful to identify the sources of scalp potentials and areas that are interconnected with source areas. Frontostriatal circuits have been shown to be involved in elicitation of the ERN (Ullsperger and von Cramon, 2006), likely routed via a thalamic relay (Seifert et al., 2011). Unfortunately, at least to our knowledge, there are no lesion studies related to the FRN. FRN data from patients with lesions in cingulate cortex or basal ganglia would be extremely valuable, in particular as lesions in both regions have been independently shown to affect feedback-based learning in humans (Bellebaum et al., 2008; Camille et al., 2011; Seifert et al., 2011). Recent findings in patients with implanted depth electrodes in VS support the notion that MCC triggers VS activation (Cohen et al., 2012).

\section{Processing of positive and negative feedback}

Discrepancies between results from fMRI and EEG studies on feedback processing in humans have accumulated during recent years (e.g., Nieuwenhuis et al., 2005; Martin et al., 2009; Carlson et al., 2011). Inspired by a theoretical framework stemming from research on the reward circuit (Haber and Knutson, 2010), the former usually report excess activation of positive relative to negative feedback, whereas the latter often assume positive feedback to be the baseline for negative feedback events (Holroyd and Coles, 2002). However, recent research has emphasized the role of positive, rewarding feedback in elicitation of the FRN/FRP in trial-averaged data (Baker and Holroyd, 2011; Carlson et al., 2011; Foti et al., 2011; Kreussel et al., 2012; Bress and Hajcak, 2013), and our approach might prove useful for distinguishing the relative contributions of rewards and nonrewards to gradations of feedback-related scalp potentials in other protocols.

As the FRN has been shown to become larger with less frequent feedback, we successfully equalized feedback presentation frequencies across conditions in this protocol. Yet, because potentials in the time range of the FRN seem to be based on overlapping contributions from the feedback-related positivity and a negativity corresponding to the N2 (Holroyd et al., 2008; Carlson et al., 2011; Foti et al., 2011), alternative designs, which elicit a stronger N2, might result in changed FRN/FRP morphology and be ultimately coupled to different hemodynamic responses. Under such conditions, a rarely occurring feedback might be perceived as more surprising. The relative infrequency of negative feedback compared with other events, including positive feedback in reversal tasks, might constitute such a case. Furthermore, tracking positive feedback over an extended time span changes the motivational value of positive feedback. This would explain findings from an EEG-fMRI study of the FRN in a reversal task (Hauser et al., 2013), which reported coupling between MCC activation and the FRN but could not find VS activation possibly because positive feedback was not motivationally salient and rewarding. Although our study controls for this aspect of FRN/FRP operationalization, one possible confounding factor is the administration of ambiguous feedback: negative and ambiguous feedback might be perceived as unequivocally negative and the administration of ambiguous feedback might therefore inflate the rate of negative feedback to $66 \%$. However, rating data on valence and arousal of both feedback types differ significantly between ambiguous and negative feedback, and this finding is difficult to reconcile with the assumption that negative and ambiguous feedback are equivalent. As negative and ambiguous feedback differ with regard to their classification by subjects, one has no reason to assume inflated expectancy of negative feedback relative to positive feedback in this design. Accordingly, the amplitudes of feedback-related potentials should not have been biased by stimulus probability effects. Further, in our implementation of the time-estimation task, considerable care has been taken to establish that the local probability of feedback information was based adaptively on response accuracy. Accordingly, positive and negative feedback are equally informative and relevant, and both are needed to clarify the response outcome.

In conclusion, converging evidence from EEG and fMRI data suggest that the FRN/FRP is tightly coupled to activation of reward-related regions, in particular VS, and medial prefrontal regions, including cingulate areas. Further, FRN/FRP seems to reflect in part a reward positivity, as activation in these areas increased as amplitudes became more positive specifically during positive feedback.

\section{References}

Allen PJ, Polizzi G, Krakow K, Fish DR, Lemieux L (1998) Identification of EEG events in the MR scanner: the problem of pulse artifact and a method for its subtraction. Neuroimage 8:229-239. CrossRef Medline

Allen PJ, Josephs O, Turner R (2000) A method for removing imaging artifact from continuous EEG recorded during functional MRI. Neuroimage 12:230-239. CrossRef Medline

Amiez C, Neveu R, Warrot D, Petrides M, Knoblauch K, Procyk E (2013) The location of feedback-related activity in the midcingulate cortex is predicted by local morphology. J Neurosci 33:2217-2228. CrossRef Medline

Baker TE, Holroyd CB (2011) Dissociated roles of the anterior cingulate cortex in reward and conflict processing as revealed by the feedback errorrelated negativity and N200. Biol Psychol 87:25-34. CrossRef Medline

Bellebaum C, Koch B, Schwarz M, Daum I (2008) Focal basal ganglia lesions are associated with impairments in reward-based reversal learning. Brain 131:829-841. CrossRef Medline

Bress JN, Hajcak G (2013) Self-report and behavioral measures of reward sensitivity predict the feedback negativity. Psychophysiology 50:610-616. CrossRef Medline

Camille N, Tsuchida A, Fellows LK (2011) Double dissociation of stimulusvalue and action-value learning in humans with orbitofrontal or anterior cingulate cortex damage. J Neurosci 31:15048-15052. CrossRef Medline

Carlson JM, Foti D, Mujica-Parodi LR, Harmon-Jones E, Hajcak G (2011) Ventral striatal and medial prefrontal BOLD activation is correlated with reward-related electrocortical activity: a combined ERP and fMRI study. Neuroimage 57:1608-1616. CrossRef Medline

Cohen MX, Cavanagh JF, Slagter HA (2011a) Event-related potential activity in the basal ganglia differentiates rewards from nonrewards: temporospatial principal components analysis and source localization of the feedback negativity: commentary. Hum Brain Mapp 32:2270-2271. CrossRef Medline

Cohen MX, Wilmes K, Vijver Iv (2011b) Cortical electrophysiological network dynamics of feedback learning. Trends Cogn Sci 15:558-566. CrossRef Medline

Cohen MX, Bour L, Mantione M, Figee M, Vink M, Tijssen MA, van Rootselaar AF, van den Munckhof P, Schuurman PR, Denys D (2012) Topdown-directed synchrony from medial frontal cortex to nucleus accumbens during reward anticipation. Hum Brain Mapp 33:246-252. CrossRef Medline

Debener S, Ullsperger M, Siegel M, Fiehler K, von Cramon DY, Engel AK (2005) Trial-by-trial coupling of concurrent electroencephalogram and functional magnetic resonance imaging identifies the dynamics of performance monitoring. J Neurosci 25:11730-11737. CrossRef Medline

Debener S, Strobel A, Sorger B, Peters J, Kranczioch C, Engel AK, Goebel R (2007) Improved quality of auditory event-related potentials recorded 
simultaneously with 3-T fMRI: removal of the ballistocardiogram artefact. Neuroimage 34:587-597. CrossRef Medline

Doñamayor N, Marco-Pallarés J, Heldmann M, Schoenfeld MA, Münte TF (2011) Temporal dynamics of reward processing revealed by magnetoencephalography. Hum Brain Mapp 32:2228-2240. CrossRef Medline

Forman SD, Cohen JD, Fitzgerald M, Eddy WF, Mintun MA, Noll DC (1995) Improved assessment of significant activation in functional magnetic resonance imaging (fMRI): use of a cluster-size threshold. Magn Reson Med 33:636-647. CrossRef Medline

Foti D, Weinberg A, Dien J, Hajcak G (2011) Event-related potential activity in the basal ganglia differentiates rewards from nonrewards: temporospatial principal components analysis and source localization of the feedback negativity. Hum Brain Mapp 32:2207-2216. CrossRef Medline

Gehring WJ, Willoughby AR (2002) The medial frontal cortex and the rapid processing of monetary gains and losses. Science 295:2279-2282. CrossRef Medline

Goebel R, Esposito F, Formisano E (2006) Analysis of functional image analysis contest (FIAC) data with brainvoyager QX: from single-subject to cortically aligned group general linear model analysis and self-organizing group independent component analysis. Hum Brain Mapp 27:392-401. CrossRef Medline

Haber SN, Knutson B (2010) The reward circuit: linking primate anatomy and human imaging. Neuropsychopharmacology 35:4-26. CrossRef Medline

Hauser TU, Iannaccone R, Stampfli P, Drechsler R, Brandeis D, Walitza S, Brem S (2013) The feedback-related negativity (FRN) revisited: new insights into the localization, meaning and network organization. Neuroimage 84C:159-168. CrossRef Medline

Holroyd CB, Coles MG (2002) The neural basis of human error processing: reinforcement learning, dopamine, and the error-related negativity. Psychol Rev 109:679-709. CrossRef Medline

Holroyd CB, Nieuwenhuis S, Yeung N, Nystrom L, Mars RB, Coles MG, Cohen JD (2004) Dorsal anterior cingulate cortex shows fMRI response to internal and external error signals. Nat Neurosci 7:497-498. CrossRef Medline

Holroyd CB, Pakzad-Vaezi KL, Krigolson OE (2008) The feedback correctrelated positivity: sensitivity of the event-related brain potential to unexpected positive feedback. Psychophysiology 45:688-697. CrossRef Medline

Huster RJ, Wolters C, Wollbrink A, Schweiger E, Wittling W, Pantev C, Junghofer M (2009) Effects of anterior cingulate fissurization on cognitive control during stroop interference. Hum Brain Mapp 30:1279-1289. CrossRef Medline

Jung J, Jerbi K, Ossandon T, Ryvlin P, Isnard J, Bertrand O, Guénot M, Mauguière F,Lachaux JP (2010) Brain responses to success and failure: direct recordings from human cerebral cortex. Hum Brain Mapp 31: 1217-1232. CrossRef Medline

Kreussel L, Hewig J, Kretschmer N, Hecht H, Coles MG, Miltner WH (2012) The influence of the magnitude, probability, and valence of potential wins and losses on the amplitude of the feedback negativity. Psychophysiology 49:207-219. CrossRef Medline

Levy DJ, Glimcher PW (2012) The root of all value: a neural common currency for choice. Curr Opin Neurobiol 22:1027-1038. CrossRef Medline

Liu X, Hairston J, Schrier M, Fan J (2011) Common and distinct networks underlying reward valence and processing stages: a meta-analysis of functional neuroimaging studies. Neurosci Biobehav Rev 35:1219-1236. CrossRef Medline
Luu P, Tucker DM, Derryberry D, Reed M, Poulsen C (2003) Electrophysiological responses to errors and feedback in the process of action regulation. Psychol Sci 14:47-53. CrossRef Medline

Martin LE, Potts GF, Burton PC, Montague PR (2009) Electrophysiological and hemodynamic responses to reward prediction violation. Neuroreport 20:1140-1143. CrossRef Medline

Miezin FM, Maccotta L, Ollinger JM, Petersen SE, Buckner RL (2000) Characterizing the hemodynamic response: effects of presentation rate, sampling procedure, and the possibility of ordering brain activity based on relative timing. Neuroimage 11:735-759. CrossRef Medline

Miltner WH, Braun CH, Coles MG (1997) Event-related brain potentials following incorrect feedback in a time-estimation task: evidence for a "generic" neural system for error detection. J Cogn Neurosci 9:788-798. CrossRef Medline

Müller SV, Möller J, Rodriguez-Fornells A, Münte TF (2005) Brain potentials related to self-generated and external information used for performance monitoring. Clin Neurophysiol 116:63-74. CrossRef Medline

Nieuwenhuis S, Holroyd CB, Mol N, Coles MG (2004) Reinforcementrelated brain potentials from medial frontal cortex: origins and functional significance. Neurosci Biobehav Rev 28:441-448. CrossRef Medline

Nieuwenhuis S, Slagter HA, von Geusau NJ, Heslenfeld DJ, Holroyd CB (2005) Knowing good from bad: differential activation of human cortical areas by positive and negative outcomes. Eur J Neurosci 21:3161-3168. CrossRef Medline

Potts GF, Martin LE, Burton P, Montague PR (2006) When things are better or worse than expected: the medial frontal cortex and the allocation of processing resources. J Cogn Neurosci 18:1112-1119. CrossRef Medline

Ruchsow M, Grothe J, Spitzer M, Kiefer M (2002) Human anterior cingulate cortex is activated by negative feedback: evidence from event-related potentials in a guessing task. Neurosci Lett 325:203-206. CrossRef Medline

Rushworth MF, Noonan MP, Boorman ED, Walton ME, Behrens TE (2011) Frontal cortex and reward-guided learning and decision-making. Neuron 70:1054-1069. CrossRef Medline

Seifert S, von Cramon DY, Imperati D, Tittgemeyer M, Ullsperger M (2011) Thalamocingulate interactions in performance monitoring. J Neurosci 31:3375-3383. CrossRef Medline

Talairach J, Tournoux P (1988) Co-planar stereotaxic atlas of the human brain. Stuttgart: Thieme.

Ullsperger M, von Cramon DY (2003) Error monitoring using external feedback: specific roles of the habenular complex, the reward system, and the cingulate motor area revealed by functional magnetic resonance imaging. J Neurosci 23:4308-4314. Medline

Ullsperger M, von Cramon DY (2006) The role of intact frontostriatal circuits in error processing. J Cogn Neurosci 18:651-664. CrossRef Medline

van Veen V, Holroyd CB, Cohen JD, Stenger VA, Carter CS (2004) Errors without conflict: implications for performance monitoring theories of anterior cingulate cortex. Brain Cogn 56:267-276. CrossRef Medline

Vogt BA (2009) Cingulate neurobiology and disease. New York: Oxford UP.

Walsh MM, Anderson JR (2012) Learning from experience: event-related potential correlates of reward processing, neural adaptation, and behavioral choice. Neurosci Biobehav Rev 36:1870-1884. CrossRef Medline

Yao J, Dewald JP (2005) Evaluation of different cortical source localization methods using simulated and experimental EEG data. Neuroimage 25: 369-382. CrossRef Medline 